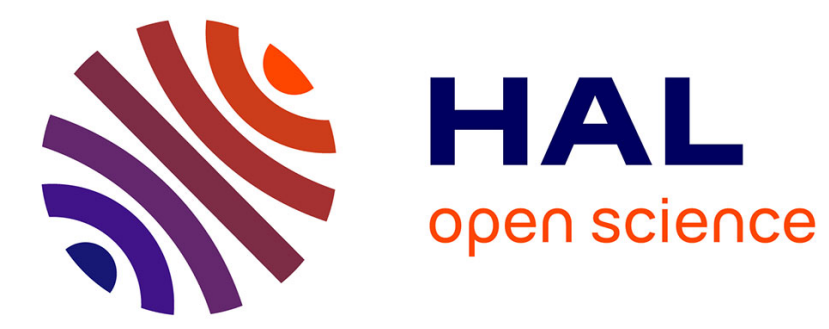

\title{
LA CRÉATION DE LABORATOIRES VÉTÉRINAIRES EN BULGARIE. Leur objet. Leur nécessité
}

K. Katrandjieff

\section{- To cite this version:}

K. Katrandjieff. LA CRÉATION DE LABORATOIRES VÉTÉRINAIRES EN BULGARIE. Leur objet. Leur nécessité. Le Lait, 1929, 9 (87), pp.743-749. hal-00894961

\section{HAL Id: hal-00894961 https://hal.science/hal-00894961}

Submitted on 1 Jan 1929

HAL is a multi-disciplinary open access archive for the deposit and dissemination of scientific research documents, whether they are published or not. The documents may come from teaching and research institutions in France or abroad, or from public or private research centers.
L'archive ouverte pluridisciplinaire HAL, est destinée au dépôt et à la diffusion de documents scientifiques de niveau recherche, publiés ou non, émanant des établissements d'enseignement et de recherche français ou étrangers, des laboratoires publics ou privés. 
cette précaution le vin prend un goût résiné et les bons vins sont particulièrement exposés à cette altération.

KUHN recommande même de pratiquer le chauffage des vins sous une pression supérieure à la pression atmosphérique pour permettre au vin de conserver tous les gaz qu'il renferme dans le foudre d'où on le soutire.

Or cette circonstance, ainsi que l'absence d'aération se trouvent précisément réalisées lorsqu'on pratique la pasteurisation en circulation continue de n'importe quel liquide, lait, vin ou jus de fruits, par le procédé de la couche mince et en utilisant comme moyen de propulsion la pompe à engrenage.

\title{
LA GRÉATION DE LABORATOIRES VÉTÉRINAIRES EN BULGARIE
}

\section{Leur objet. - Leur nécessité}

\author{
par K. KATRANDJEFF
}

Chef de Service à l'Institut Bactériologique Vétérinaire de Sofia

Lorsque Bourgelat fonda à Lyon la première Ecole Vétérinaire, sans doute pensait-il à créer un lien entre la médecine humaine et la médecine des animaux, celle-là devant puiser chez celle-ci des comparaisons utiles, des exemples féconds, mais il ne pouvait déjà se douter du grand rôle que la médecine vétérinaire aurait à jouer plus tard dans l'hygiène publique.

Il est indubitable que la solidité de l'armature de l'enseignement vétérinaire met aujourd'hui notre confrère à même d'intervenir d'une façon opportune, d'une part, dans la sauvegarde de l'hygiène publique, d'autre part, dans toutes les industries qui se rapportent aux productions animales. II y a d'ailleurs un lien très étroit entre l'un et l'autre de ces points, puisque l'alimentation humaine emprunte considérablement aux animaux, qu'il s'agisse de la viande, apprêtée ou non, qu'il s'agisse du lait et des dérivés de celui-ci.

Depuis la guerre, les animateurs du Service vétérinaire en Bulgarie se sont aperçus eombien il était utile, nécessaire même, de développer dans ce pays tout ce qui se rapporte aux industries animales, mais en même temps, et la question s'imposait évidemment à leur attention, ils ont senti que rien de bien ne pouvait être fait dans cette direction sans être aecompagné d'un contrôle hygiénique efficace de tous les produits qui s'y rapportent.

En Bulgarie, l'organisation des services vétérinaires est telle que l'on peut concevoir la constitution d'un cadre solide de fonctionnaires éclairés chargés du susdit contrôle.

Il ne faut point oublier que la Bulgarie est un Etat uniquement 
agricole, tirant donc de la seule surface de son sol toutes ses richesses. Il faut que celles-ci se développent en vue du meilleur rendement et de la meilleure qualité.

Un phénomène économique d'une très grande importance se constate dans tous les pays provenant d'anciens empires et auxquels le traité de Versailles a donné la liberté. Ce phénomène se traduit par le souci qu'ont les dirigeants de ces nouvelles nations de donner une estampille officielle aux produits agricoles que l'on voudrait voir se répandre sur tous les marchés mondiaux. C'est surtout dans les produits laitiers, plus facilement étalonnables, que cette préoccupation se manifeste; mais elle peut s'étendre également à d'autres productions animales.

Ce système $a \mathrm{du}$ bon parce que les garanties officielles assurent une régularité de qualité qui est à la base du développement des transactions portant sur les matières alimentaires.

Dans ces conditions, les Etats n'hésitent pas à dépenser pour créer des laboratoires de contrôle, entretenir un cadre de fonctionnaires utiles, sachant très bien que ce ne sont là après tout que des avances.

La Bulgarie est entrée dans le mouvement que nous venons de signaler. Des lois, des règlements dont la rédaction semble répondre aux exigences modernes, ont été édictés en vue de l'amélioration des industries animales. C'est ainsi que successivement on a vu décréter :

10 La tuberculination obligatoire des vaches laitières ;

$2^{\circ}$ Le contrôle sanitaire vétérinaire des femelles productrices de lait;

$3^{\circ}$ Le contrôle de la construction des étables modernes;

4. Le contrôle vétérinaire de l'approvisionnement des villes en lait et en produits dérivés de ce dernier ainsi que celui de leur vente ;

$5^{\circ}$ Le contrôle vétérinaire de la construction des fromageries et des produits qu'elles fabriquent.

Incontestablement, il y a là un ensemble de lois du plus haut intérêt. Toutefois, il est toujours une marge très grande entre les textes et leur application. Cela est vrai pour tous les pays, mais plus encore peut-être pour la Bulgarie, parce que le cadre des vétérinaires qui doit fournir les agents d'exécution n'est pas encore au complet. J'ajouterai que des raisons techniques s'opposent pour l'instant à voir jouer, du moins pour tout le pays, ces lois et les règlements qui en découlent comme le voudrait un hygiéniste éelairé.

En Bulgarie, le service vétérinaire est service d'Etat. Il est organisé comme suit : un service central au Ministère d'Agrieulture avee son directeur qui est le chef et trois inspecteurs généraux.

Les vétérinaires d'Etat comprennent des vétérinaires départementaux, d'arrondissements et de petites régions. Tous ont à s'occuper du traitement des maladies courantes, de la clinique, en d'autres termes, de la lutte contre les épizooties et de l'amélioration de l'élevage.

Le contrôle des denrées alimentaires d'origines animales et la lutte 
contre la tuberculose sont dans les attributions du vétérinaire communal, sous l'impulsion du service central (Ministère d'Agriculture). Là où il n'y a pas de vétérinaire communal la susdite tâche est confiée au vétérinaire d'arrondissement ou au vétérinaire régional.

La difficulté des communications en certains points du territoire, le nombre trop faible encore des vétérinaires rendent incomplète la parfaite application des règlements, et l'on constate souvent que l'homme de l'art risque d'arriver trop tard pour prendre les mesures utiles qui s'imposent.D'autre part le vétérinaire qui est le conseil-né de l'agriculteur en ce qui concerne la production animale et toutes les industries qui s'y rattachent, se trouve trop souvent dans l'impossibilité de remplir son rôle au mieux des intérêts de la nation parce qu'il exerce ses fonctions sur un rayon trop étendu. Ses efforts sont disséminés, partant, ils ne peuvent pas être aussi productifs qu'on le voudrait. Il s'impose done de voir augmenter le nombre des vétérinaires, mais cela ne saurait suffire. Il faut également, et même avant tout, que les vétérinaires qui sont en contact permanent avec les milieux agricoles, intéressés plus particulièrement aux productions animales, puissent non seulement trouver dans des laboratoires bien outillés les enseignements utiles, mais soient aussi dans l'obligation de s'y rendre périodiquement pour s'initier aux nouvelles méthodes dont l'application peut faciliter leur tâche quotidienne.

Tout se tient dans les questions que nous agitons ici. Derrière l'Hygiène publique avec ses exigences, nous voyons l'Economie laitière présenter également les siennes. La création de laiteries coopératives est souhaitable. On peut en prévoir le développement rapide dans un pays comme la Bulgarie, mais pour cela faut-il encore que l'impulsion qui en sera donnée s'inspire de tout ce qui se fait à l'étranger, là surtout où le développement coopératif a augmenté la richesse, afin d'éviter les mécomptes et les erreurs. En une pareille matière, il n'y a point à innover; il n'y a simplement qu'à imiter les bons exemples qui ne manquent pas.

Pour alimenter les laiteries coopératives qui auront à fournir du bon lait, du beurre et des fromages d'excellente qualité, il faut évidemment la possession d'une matière première irréprochable. En Bulgarie, nous sommes très bien placés pour viser à ce but, en raison même de l'esprit qui anime le service vétérinaire, lequel, comme je le disais un peu plus haut, est un service d'Etat.

En Bulgarie, existe, et nous l'avons dit déjà, le contrôle sanitaire vétérinaire des animaux laitiers, dans lesquels, à côté de la vache, il faut placer la bufflesse, la chèvre et la brebis. Mais, en ne nous en tenant qu'aux deux premières de ces femelles, nous n'ignorons pas combien il y a à lutter contre les maladies de leur mamelle. On ne saurait sousestimer le préjudice considérable causé par les infections mammaires à la production laitière. La question ne frappe l'esprit des gens qui y 
sont intéressés par fonstion qu'en période d'épidémies a phteuses notam. ment, car la situation est aigüe à ce moment. Mais lorsque l'épidémie disparaît, on ne voit pas assez les séquelles qu'elle laisse et dont les infections mammaires sont incontestablement les plus importantes.

- On ne saurait en conscience s'opposer à l'établissement d'une police sanitaire des mamelles visant d'un côté, au traitement des mammites subaigües et chroniques qui font tant de tort à la production laitière, et de l'autre à l'éradication des femelles laitières dont la glande fortement touchée ne doit plus être entretenue en vue d'en retirer du lait; l'animal ne " payant " plus de ce côté, doit être sans retard destiné à la boucherie.

Il importe d'insister sur la fréquence de l'intervention vétérinaire sur un pareil terrain. Elle donnera les résultats les plus heureux, puisque mes confrères seront au courant de tous les procédés qui leur permettront de dézeler à coup sûr l'atteinte de la mamelle, d'en mesurer l'étendue, par suite de se mettre mieux à même d'en combattre les conséquences.

En Bulgarie, disions-nous encore, la construction des fromageries est placée sous la surveillance des vétérinaires, mais le contrôle jusqu'à aujourd'hui se limite au point de vue hygiénique. Or, s'il y a de ce côté un effort considérable à réaliser à tous les stades de la route que le lait doit suivre, depuis la mamelle jusqu'au consommateur chez qui il arrive sous les aspects les plus variés : lait en nature, beurre et fromage, il est indiscutable qu'un perfectionnement doit être apporté aux méthodes de production et de manipulation du lait si l'on veut donner aux produits bulgares une réputation de bonne qualité à laquelle nous pouvons prétendre comme bien d'autres pays d'ailleurs.

Il faut que l'industrie laitière bulgare, dans quelque direction qu'on l'envisage, soit améliorée, modernisée, et pour ee, soit placée sous la surveillance constante du service vétérinaire. Cela lui permettra, encore une fois, de se faire une place honorable sur les marchés étrangers, spécialement en Turquie, pays voisin très à sa portée, pour absorber l'excédent de sa production nationale.

L'estampille officielle des produits destinés à l'exportation est une conséquence toute naturelle de cette surveillance.

A l'heure actuelle, l'industrie laitière en Bulgarie a conservé, sauf de rares exceptions, son caractère primitif. Toutefois, il y a lieu de noter la tendance à la voir perdre son importance purement locale. La production laitière est essentiellement disséminée, dispersée. Les producteurs sont légion, mais il ne doit pas en être de même du travail de la matière première. Los petites industries locales qui s'ingénient à faire beurre et fromages, sans méthode, sans grand soin, n'aboutissent pas à des produits d'une qualité régulière. Il est im possible, a vec un tel système économique, de prétendre à la conquête des marchés étrangers. La production doit être concantrée, pour qu'elle soit travaillée de la meilleure façon. L'exem. ple des pays chez lesquels l'industrie laitière est source de richesse natio- 
nale est là pour nous le montrer. Qu'il s'agisse du beurre comme en Dane. mark par exэmple, du fromage comme en Hollande, en Finlande, etc., on constate que la réputation des produits venant de ces pays est la conséquense toute naturelle de la concentration du lait en grandes quantités dans des laiteries parfaitement outillées pour le travailler.

En Bulgarie, non seulement nous devons envisager le lait de vache, mais également eelui de bufflesse qui au point de vue économique joue un rôle extrêmement important dans l'industrie nationale. Comme le lait de vache, il tombe sous la surveillance des vétérinaires, mais là, plus peut-être qu'avec l'autre lait, l'intervention des services compétents doit être particulièrement énergique.

Les fromages de Bulgarie commencent à être connus, et des travaux scientifiques du meilleur aloi ont été faits sur leur technique et leur flore microbienne. Un progrès s'appuyant sur ces derniers, et sur toutes les acquisitions de la science fromagère, - qu'elles s'appliquent à telle ou telle espèce de fromages, il importe peu, car il est des règles générales qui doivent être observées dans tous les cas, - conduira à n'en pas douter à l'obtention d'un produit régulier, standardisé que l'estampille officielle couvrira toutes les fois que la qualité sera bonne.

A côté des fromages, il est également le beurre. Ici encore, l'introduction des méthodes modernes s'impose, tant pour la production que pour la vente. L'écrémage, la pasteurisation des crèmes et l'ensemencement de celles-ci, le contrôle du produit fabriqué, la standardisation, tout cela doit être réalisé sous l'impulsion du service vétérinaire. Notre confrère exercera son rôle hygiénique, il guidera également la technique et permettra ainsi d'écouler sur les marchés consommateurs des produits excellents.

Je ne parlerai pas ici des falsifications, car cette question découle tout naturellement des considérations que je viens de faire valoir. De fait, un contrôle de la production, de la technique et de la vente, comme il est facile de le prévoir, facilite la lutte contre la fraude. On peut même a vancer à coup sûr que la méthode préventive qui doit jouer ici s'opposera à son développement, mais l'ingéniosité de l'homme est telle, lorsqu'il s'agit de frauder, qu'une surveillance très attentive est toujours nécessaire, surtout, encore une fois, si nous avons la prétention en Bulgarie d'ameliorer nos produits de telle façon que nous puissions viser la conquête des marchés étrangers.

Pour que les vétérinaires soient tout à fait à même de répondre à la haute mission que leur dictent les lois et que nous voudrions leur voir remplir aveo la plus parfaite compétence, il est indispensable que des laboratoires tort bien outillés soient créés pour leur permettre de les mettre au courant de toutes les applications de la science susceptibles de leur rendre des services.

J'ai déjà abordé ce point un peu plus haut, mais je dois maintenant 
m'expliquer sur la façon dont on doiţ comprendre, à mon avis, l'organisation et le fonctionnement de ces laboratoires.

Aujourd'hui et de plus en plus, le vétérinaire praticien s'adresse aux laboratoires pour éclairer son diagnostic, guider ses interventions, dicter sa thérapeutique.

En Bulgarie où le vétérinaire est fonctionnaire, il sera dans l'obligation de recourir régulièrement aux laboratoires prévus. Les nouveaux prosédés de tuberculination, la lutte contre l'avortement épizootique, l'emploi dos méthodes si variées de tendance et de discipline nécessaires à connaître pour lutter à bon escient contre les mammites, l'examen des produits de l'industrie animale, n'y a-t-il pas là tout un ensemble de faits exigeant que le vétérinaire soit en contact permanent avec le labo. ratoire. Isolé, abandonné à lui-même, dans le fond des campagnes, sans aucune obligation pour lui de fréquenter un laboratoire vétérinaire où il serait aussitôt éslairé sur les questions qui l'intéressent, il n'est pas capable toujours de remplir sa mission comme le veut impérativement le souci de l'hygiène publique.

En Bulgarie rien n'est plus simple. Notre confrère fréquentera le laboratoire vétérinaire régional où très rapidement il sera mis au courant des questions qui lui seront utiles. Et quand il rentrera dans le cercle de son activité, ce sera un homme mieux éclairé, plus averti qui sera appelé à faire də la bonne police sanitaire envisagée dans son sens le plus large. Et si nous revenons sur la question des mammites, il s'emploiera à dépister les atteintes les plus légères du parenchyme glandulaíre au lieu d'attendre de se trouver en face d'infections massives plus ou moins explosives contre lesquelles il est plus difficile d'intervenir.

Au-dessus des laboratoires départementaux, il y aurait lieu de voir édifier en Bulgarie un Institut de laiterie plus particulièrement destiné à la rechershe. Lo laboratoire régional près duquel le vétérinaire viendra périodiquement se renseigner est là pour l'aider dans l'accomplissement de sa mission quotidienne; car il donne les conseils, fait les diagnostics, oriente la police sanitaire, etc., mais il n'aura pas le temps de se lancer dans une série de recherches nouvelles. C'est l'Institut central qui aura ce soin. Ses services de chimie, de bactériologie, de physique auront le pərsonnel et le matériel voulus. Il leur sera donc possible d'étudier les questions que l'intérêt ésonomique de la Bulgarie fera naître, de répondre aussi aux suggestions de l'industrie laitière. Il y a là un large champ à exploiter. C'est de l'Institut central que se déversera vers les la boratoires départem ntaux la mise au point des instructions nouvelles à donner au cadra des vétérinaires de l'Etat. C'est à l'Institut central également que səront étudiés les projets de construction d'étables, de beurreries, de fromageries, d'usines de pasteurisation, etc.

Je n'insiste pas davantage sur l'utilité d'un tel organisme. Elle va de soi. Mais en terminant, je reviendrai sur ce point à nouveau, c'est que la 
Bulgarie, pays essentiellement agricole ne doit pas oublier que toutes les dépenses faites pour l'amélioration des industries animales seront remboursées largement par l'extension de ses marchés extérieurs. Les vétérinaires ont ici à jouer ur rôle dont ils comprennent tous les premiers la haute importance.

\section{LA VITAMINE C DANS LE LAIT CONCENTRÉ SUCRÉ}

Par Dr A. BAKKE

Chef des Services scientifiques de la Nestlé et Anglo-Swiss Condensed Milk Co.

Assisté par Mile V. ASCHEHOUG

Assistante au laboratoire de recherches de la Nestlé et Anglo-Swiss Cond. Milk Co.

Parmi les vitamines les plus connues (nous parlons des vitamines A, B, C et D), c'est la vitamine C qui est la plus fragile. On peut dire que toutes les vitamines sont plus thermostables qu'on ne l'avait considéré au début de l'étude de ces substances ou principes. On sait aujourd'hui que les conditions dans lesquelles agit la chaleur, notamment le milieu, et surtout l'oxydation, jouent un rôle essentiel dans la résistance des vitamines à la chaleur. Ceci s'applique également à la durée de leur conservation. Il n'en reste pas moins 'que la vitamine " $\mathrm{C}$ » est la plus fragile de toutes. Il est donc particulièrement intéressant d'examiner comment elle se comporte dans les conserves de lait et surtout dans le lait concentré.

Il y a deux grandes elasses de lait concentré. L'une, comprend uniquement le lait concentré sucré, dont la conservation est assurée uniquement par l'extraction de la plus grande partie de l'eau qui entre dans sa composition et par l'adjonction d'une quantité convenable de saccharose. Le lait con:entré sucré n'est pas stérilisé.

L'autre classe comprend essentiellement les laits stérilisés non sucrés, concentrés ou non, et dont la conservation est assurée par une stérilisation thermique rigoureuse, qui doit, pour atteindre son but, détruire absolument tous les germes contenus dans le lait. sucré.

Le produit qui nous intéresse dans ce travail est le lait concentré

Pour nos essais, nous avons utilisé des cobayes, quoique certains auteurs estiment que la sensibilité des animaux à l'absence de vitamines "C $\mathrm{C}$ est trop grande pour qu'il soit possible d'en tirer des conclusions applicables à l'alimentation humaine.

Cependant, comme il existe des raisons de croire que le rôle de la vitamine " $\mathrm{C}$ " ne se limite pas à une action antiscorbutique, mais agit 